Bentham OPEN
CrossMark
$\begin{aligned} & \text { Content list available at: www.benthamopen.com/TOOPHTJ/ } \\ & \text { DOI: } 10.2174 / 1874364101711010211\end{aligned}$

REVIEW ARTICLE

\title{
Collagen Cross- Linking for Paediatric Keratoconus
}

Georgios D. Panos ${ }^{1, *}$, Nikolaos Kozeis², Miltiadis Balidis ${ }^{2}$, Marilita M. Moschos ${ }^{3}$ and Farhad Hafezi $^{4}$

${ }^{I}$ Department of Ophthalmology, Ipswich Hospital NHS Trust, University of Cambridge, Ipswich, Suffolk, UK

${ }^{2}$ Ophthalmica Institute, Thessaloniki, Central Macedonia, Greece

${ }^{3}$ Department of Ophthalmology, School of Medicine, National and Kapodistrian University of Athens, Greece

${ }^{4}$ ELZA Institute, Zurich, Switzerland; Faculty of Medicine, University of Geneva, Geneva, Switzerland; Faculty of Medicine, University of South California, California, USA

Received: January 14, 2017

Revised: May 13, 2017

Accepted: June 13, 2017

\section{Abstract:}

\section{Background:}

Since the late 1990s corneal crosslinking (CXL) has been proposed as a new treatment option which can stop progression of keratoconus with promising results in adults.

\section{Objective:}

Keratoconus presents a higher rate and faster progression in paediatric patients and for this reason prompt and effective treatment is essential. Due to its success in adult keratoconus patients, CXL has been recently applied to children in order to stop or slow progression of keratoconus in paediatric patients.

\section{Conclusions:}

This article will present an update of the literature on the topic of CXL in this age group.

Keywords: Paediatric keratoconus, Collagen cross- linking, Biometry, Cornea.

\section{INTRODUCTION}

Keratoconus is a progressive, bilateral, and asymmetric non-inflammatory corneal ectasia [1]. The disease traditionally manifests in the $2^{\text {nd }}$ decade of life when the cornea presents an increasingly conical shape, secondary to its biomechanical instability, which leads to irregular astigmatism and subsequent reduced visual acuity [1 - 3].

Traditional management of keratoconus consists of visual rehabilitation by means of spectacles, contact lenses and intracorneal ring implants for early to moderate stages and lamellar or penetrating keratoplasty in advanced stages $[1,4-6]$.

The introduction of corneal collagen cross-linking (CXL) by Wollensak et al. has changed the management of keratoconus [7]. CXL is a technique that uses the photochemical reaction between the Ultraviolet A (UVA) light and riboflavin within the corneal stroma and leads to the development of chemical bonds between collagen fibrils strengthening the cornea and slows or stops the progression of keratoconus and other corneal ectasia [8,9]. Following its success in adult patients, CXL has been recently used for the treatment of paediatric patients with keratoconus [10 - 14].

\footnotetext{
* Address correspondence to this author at the Eye Department, The Ipswich Hospital NHS Trust, Heath Road, IP4 5PD, Ipswich, Suffolk, UK, Tel: +44 (0) 1473 712233; Fax: +44 (0) 1473 703400; E-mails: gdpanos@gmail.com; gdpanos@yahoo.gr
} 


\section{CXL PROCEDURE}

Standard CXL as described by Wollensak et al. is performed after removal of the central 7-9 mm of the epithelium using isotonic riboflavin $0.1 \% 20 \%$ and dextran solution every 5 minutes for 30 minutes. Ultraviolet-A irradiation (370 $\mathrm{nm}, 3 \mathrm{~mW} / \mathrm{cm}^{2}$ ) is performed during 30 minutes and isotonic riboflavin solution is re-applied every 5 minutes. Finally, a bandage lens is placed and oral pain medication and antibiotic eye drops are prescribed [7]. Since, this first report there is a large number of publications in the literature reporting safety and efficacy of CXL in the treatment of keratoconus

and other corneal ectatic conditions (ex. pellucid marginal degeneration) [15 - 19].

These studies have provided evidence that CXL is effective in slowing or stopping keratoconus progression and may even improve patients vision by inducing corneal flattening and reduction in irregular astigmatism. Moreover, CXL (epithelium-off Dresden protocol) was found to be safe for corneal endothelium and intra-ocular structures when inclusion criteria are fulfilled (corneal thickness at least $400 \mathrm{um}$ ) with an acceptable rate of complications [15, 16].

Apart from the standard epithelium-off Dresden protocol, some clinicians have elected to perform CXL with the epithelium intact or partially disrupted or with the use of femtosecond-created intrastromal pockets, in an attempt to reduce post-operative discomfort and accelerate visual recovery [20 - 22]. The use of repeated applications of tetracaine $1 \%$ to try to loosen epithelial tight junctions has also been described [20]. Clinical studies have shown encouraging results however it remains controversial whether these novel protocols are as efficient as the standard epithelium-off one [23 - 27].

Standard treatment protocol utilizes UVA energies of $3 \mathrm{~mW} / \mathrm{cm}^{2}$ and requires $30 \mathrm{~min}$ of UVA exposure to achieve the desired clinical effect as it is described above. It has been theorized that by increasing the UVA fluence while simultaneously reducing the exposure time, the same sub-threshold cytotoxic corneal endothelial UVA dosage can be administered, thereby maintaining efficacy and safety, but with a reduced treatment time. Cinar et al. in a study of 23 eyes showed that accelerated CXL produced a significant reduction in topographic keratometry values and an improvement in corrected distance acuity [28]. Kanellopoulos in a randomized, prospective study using a UVA power of $7 \mathrm{~mW} / \mathrm{cm}^{2}$ for $15 \mathrm{~min}$ compared to $3 \mathrm{~mW} / \mathrm{cm}^{2}$ for $30 \mathrm{~min}$ has demonstrated similar clinical results as the standard technique in terms of ectasia stabilization without any adverse effects associated with the higher fluence, shorter duration treatments [29].

\section{CXL IN PAEDIATRIC PATIENTS}

Keratoconus is most frequently diagnosed in young adults, however corneal changes (ex. ectasia) start much earlier [1]. It is well documented that keratoconus in paediatric patients presents a higher rate and speed of keratoconus progression compared to adults and is more aggressive [13, 30 - 35]. This may lead to a faster visual deterioration in this group of patients and affect the social and educational development and consequently their quality of life. Treating keratoconus at an earlier age is more beneficial than waiting until patients have more advanced disease requiring corneal transplantation. As the prognosis of corneal transplantation in children is poorer than in adults [36], a treatment to halt the progression, before corneal graft is necessary, could be of great benefit. CXL is effective and safe in halting the progression of keratoconus in adults. For this reason, CXL has been recently applied and evaluated in children.

Arora et al. [10], in their prospective study applied standard CXL in 15 eyes of 15 pediatric keratoconus patients (10 to 15 years) with moderate keratoconus in 1 eye and advanced disease in the fellow eye. At the end of the follow-up period (1 year), mean uncorrected distance visual acuity (UDVA) improved significantly from $1.00 \pm 0.30$ (20/200) to $0.72 \pm 0.29(20 / 100) \operatorname{logMAR}(\mathrm{P}=.035)$ and mean corrected distance visual acuity (CDVA) from $0.56 \pm 0.21(20 / 70)$ to $0.30 \pm 0.15(20 / 40) \operatorname{logMAR}(\mathrm{P}=.003)$. Mean change in apical $\mathrm{K}(1.01 \pm 2.40$ diopters $)$ was also significant $(\mathrm{P}=.004)$. No significant complications were noted.

Vinciguerra et al. [12], in a prospective, interventional study included 40 eyes of 40 paediatric patients which underwent CXL. Mean $\log$ MAR baseline UDVA and CDVA were $0.79 \pm 0.21$ and $0.39 \pm 0.10$, respectively. Mean UDVA and CDVA at 2 years were $0.58 \pm 0.18$ and $0.20 \pm 0.09$, respectively. The improvement for both UDVA and CDVA was significant throughout the postoperative follow-up $(\mathrm{P}<.05)$. Mean baseline simulated keratometry was 46.32 D in the flattest meridian and 51.48 D in the steepest meridian; at 2 years, the values were $45.30 \mathrm{D}(\mathrm{P}=.04)$ and $50.21 \mathrm{D}(\mathrm{P}=.07)$, respectively. Moreover, for a 3-mm pupil, there was a significant reduction $(\mathrm{P}<.05)$ in whole eye (total), corneal, higher-order, and astigmatic wavefront aberrations at 24 months. A significant difference $(\mathrm{P}<.05)$ in total coma and total spherical aberration 2 years after CXL also was observed. 
Caporrossi et al. [11], conducted a prospective nonrandomized phase II open trial (the "Siena CXL Pediatrics") involving 152 patients aged 18 years or younger (10-18 years) with a follow-up of 36 months. UDVA and CDVA increased by +0.18 and +0.16 Snellen lines respectively in the thicker group (corneal thickness $>450 \mu \mathrm{m}$ ) and +0.14 and +0.15 Snellen lines, respectively, in the thinner group (corneal thickness $<450 \mu \mathrm{m}$ ). Topographic results showed statistically significant improvement in $\mathrm{K}$ readings and asymmetry index values. Coma reduction was also significant.

Our research group conducted a retrospective study 59 eyes from 42 children and adolescents (aged 9 to 19 years) with confirmed keratoconus with up to 3 years follow up [13]. Fifty-two of the 59 eyes enrolled in this study showed progression, corresponding to a progression rate of $88 \%$. Forty-six eyes were treated by CXL. Maximal keratometry, CDVA, and KI showed significant changes over the follow-up period. However, significant Kmax reduction observed up to 24 months after CXL lost significance at 36 months. They proposed that awaiting documentation of progression is not mandatory and CXL in children and adolescents should be performed as soon as the diagnosis has been made. Zotta et al. in their retrospective case series evaluated the outcomes of CXL in paediatric patients with bilateral progressive keratoconus [37]. Four paediatric patients (eight eyes) with progressive keratoconus aged 14.0 \pm 2.2 years (range: 11 to 16 years) were included with a follow-up period of 36 months. All eyes underwent CXL in accordance with the standard Dresden protocol. Stabilization of K1 and K2 was demonstrated in all cases throughout follow-up while visual acuity improved in six eyes and remained stable in the remaining two eyes.

Magli et al. [23] conducted a comparative analysis of standard CXL (epi-off) and trans-epithelial CXL (TE CXL) protocols in a retrospective comparative evaluation of 37 eyes of 29 patients (12-18 years). In the epi-off CXL group (19 patients, 23 eyes; mean age, $14.75 \pm 2.1$ years), a significant improvement at month 12 was present for Kmax [-1.11 diopters (D), $\mathrm{P}=0.01]$, Kmin $(-3.2 \mathrm{D}, \mathrm{P}=0.001)$, mean $\mathrm{K}(-1.47 \mathrm{D}, \mathrm{P}=0.01)$, surface asymmetry index $(-0.64 \mathrm{D}, \mathrm{P}=$ $0.001)$, inferior-superior symmetry index $(-0.54 \mathrm{D}, \mathrm{P}=0.01)$, index of height asymmetry $(-2.97, \mathrm{P}=0.03)$, and anterior elevation at the thinnest location $(-2.82 \mathrm{D}, \mathrm{P}=0.01)$ and at the apex $(-2.27 \mathrm{D}, \mathrm{P}=0.01)$. Postoperative corneal oedema lasted 3 months in 16 eyes $(69.5 \%)$ and more than 6 months in 2 eyes $(8.7 \%)$. In the TE-CXL group (10 patients, 14 eyes; mean age, $15 \pm 4.2$ years), a significant improvement at month 12 was present for $\operatorname{Kmax}(-1.14 \mathrm{D}, \mathrm{P}=0.02)$, Kmin (-2.04 D, P = 0.01), mean K (-1.63 D, P = 0.01), surface asymmetry index $(-0.86 \mathrm{D}, \mathrm{P}=0.001)$, inferior-superior symmetry index $(-0.55 \mathrm{D}, \mathrm{P}=0.001)$, index of height asymmetry $(-2.95, \mathrm{P}=0.01)$, and anterior elevation at the thinnest location $(-2.96 \mathrm{D}, \mathrm{P}=0.01)$ and at the apex $(-2.19 \mathrm{D}, \mathrm{P}=0.01)$. No postoperative corneal oedema after TE-CXL was observed. Changes at month 12 from baseline were not significantly different between the 2 groups $(\mathrm{P}>0.05)$. TE-CXE was significantly less painful than epithelium-off CXL.

On the other hand, Buzzonetti and Petrocelli [27], performed a prospective analysis of TE CXL for paediatric keratoconus ( 8 to 18 years age) in 13 eyes of 13 patients and demonstrated that despite CDVA improvement, transepithelial CXL does not effectively halt keratoconus progression in children compared to standard CXL.

Salman conducted a prospective, comparative study including 22 eyes of 22 patients younger than 18 years with bilateral keratoconus [38]. They had transepithelial CXL with the use of transepithelial riboflavin. The other eye was used as a control and was treated conservatively. After transepithelial CXL, the improvement in the mean UDVA was statistically significant (from $0.95 \pm 0.34 \log \mathrm{MAR}$ to $0.68 \pm 0.45 \log \mathrm{MAR})(\mathrm{P}<.05)$. No eye lost lines of preoperative UDVA; 1 eye lost 1 line of preoperative CDVA. The mean simulated keratometry $(\mathrm{K})$ decreased by a mean of 2.03 diopters (D), with mean flattening of the apical K by $2.20 \mathrm{D}$; both results were statistically significant $(\mathrm{P}<.05)$. In the control group, the simulated $\mathrm{K}$ increased by a mean of $0.59 \mathrm{D}(\mathrm{P}>.05)$, with mean steepening of the apical $\mathrm{K}$ by $2.9 \mathrm{D}$ $(\mathrm{P}<.05)$ suggesting that preliminary results of transepithelial $\mathrm{CXL}$ in children with keratoconus were encouraging, with no evidence of progression of keratoconus over 12 months.

Recently McAnena and 'Keefe published a retrospective study in order to report the visual, refractive, and tomographic outcomes of corneal collagen crosslinking (CXL) in paediatric patients with keratoconus [39]. They demonstrated that CXL effectively stabilized uncorrected visual acuity, refractive indices, and keratometry values at 1 year, while improving best-corrected visual acuity.

A summary of the studies is depicted in (Table 1).

Table 1. Summary of published studies regarding outcomes of corneal collagen cross linking in paediatric keratoconus.

\begin{tabular}{|c|c|c|c|c|c|c|}
\hline Study & Subjects & Age & Design & Follow-up & CXL Protocol & Outcome \\
\hline Arora et al. & 15 & $10-15$ & Prospective & 12 months & Standard & Improvement \\
\hline Vinciguerra et al. & 40 & $9-18$ & Prospective & 24 months & Standard & Improvement \\
\hline
\end{tabular}


(Table $\square$ ) contd.....

(Table 凹) contd.....
\begin{tabular}{|c|c|c|c|c|c|c|}
\hline Study & Subjects & Age & Design & Follow-up & CXL Protocol & Outcome \\
\hline Caporrossi et al. & 152 & $10-18$ & Prospective & 36 months & Standard & Improvement \\
\hline Chatzis et al. & 42 & $9-19$ & Retrospective & 36 months & Standard & Initial improvement, late progression \\
\hline Zotta et al. & 4 & $11-16$ & Retrospective & 36 months & Standard & Improvement or stabilization \\
\hline Magli et al. & 29 & $12-18$ & Retrospective & 12 months & Epi-on and Epi-off & Improvement for both groups \\
\hline Buzzonetti and Petrocelli & 13 & $8-18$ & Prospective & 18 months & Epi-on & Progression \\
\hline Salman & 22 & $13-18$ & Prospective & 12.05 months (mean) & Epi-on & Improvement \\
\hline McAnena and O'Keefe & 14 & $13-18$ & Petrospective & 12 months & Standard & Stabilization \\
\hline
\end{tabular}

\section{CONCLUSION}

Keratoconus presents a higher rate and faster progression in paediatric patients and for this reason prompt and effective treatment is essential. Despite limited evidence in paediatric patients, CXL may be considered in the management of progressive paediatric keratoconus when comparing the risks of visually significant complications from CXL and the risk of visual loss from accelerated progression of keratoconus in young patients, it is clear that CXL should be offered without waiting for progression. Standard epithelium-off CXL protocol should be applied, as there is no evidence yet that TE - CXL can provide similar efficiency and safety. Parents should be aware of possible adverse effects, short lasting effect and need for re-treatment in cases with aggressive form. Future research should focus on possible different protocols regarding the power and the duration of the technique, and on the timing of the treatment in order to improve long term outcomes and reduce complications. These results are anticipated with great interest.

\section{CONSENT FOR PUBLICATION}

Not applicable.

\section{CONFLICT OF INTEREST}

The authors declare no conflict of interest, financial or otherwise.

\section{ACKNOWLEDGEMENTS}

Declared none.

\section{REFERENCES}

[1] Rabinowitz YS. Keratoconus. Surv Ophthalmol 1998; 42(4): 297-319. [http://dx.doi.org/10.1016/S0039-6257(97)00119-7] [PMID: 9493273]

[2] Davis LJ, Schechtman KB, Wilson BS, et al. Longitudinal changes in visual acuity in keratoconus. Invest Ophthalmol Vis Sci 2006; 47(2): 489-500. [http://dx.doi.org/10.1167/iovs.05-0381] [PMID: 16431941]

[3] McMahon TT, Edrington TB, Szczotka-Flynn L, Olafsson HE, Davis LJ, Schechtman KB. Longitudinal changes in corneal curvature in keratoconus. Cornea 2006; 25(3): 296-305. [http://dx.doi.org/10.1097/01.ico.0000178728.57435.df] [PMID: 16633030]

[4] Bahar I, Kaiserman I, Srinivasan S, et al. Comparison of three different techniques of corneal transplantation for keratoconus. Am J Ophthalmol 2008; 146(3): 905-12. [http://dx.doi.org/10.1016/j.ajo.2008.06.034]

[5] Bromley JG, Randleman JB. Treatment strategies for corneal ectasia. Curr Opin Ophthalmol 2010; 21(4): 255-8. [PMID: 20489623]

[6] Zare MA, Hashemi H, Salari MR. Intracorneal ring segment implantation for the management of keratoconus: safety and efficacy. J Cataract Refract Surg 2007; 33(11): 1886-91 [http://dx.doi.org/10.1016/j.jcrs.2007.06.055] [PMID: 17964393]

[7] Wollensak G, Spoerl E, Seiler T. Riboflavin/ultraviolet: An induced collagen crosslinking for the treatment of keratoconus. Am J Ophthalmol 2003; 135(5): 620-7.

[http://dx.doi.org/10.1016/S0002-9394(02)02220-1] [PMID: 12719068]

[8] Suri K, Hammersmith KM, Nagra PK. Corneal collagen cross-linking: Ectasia and beyond. Curr Opin Ophthalmol 2012; 23(4): 280-7. [http://dx.doi.org/10.1097/ICU.0b013e328354865e] [PMID: 22569470]

[9] O’Brart DP. Corneal collagen cross-linking: A review. J Optom 2014; 7(3): 113-24. [http://dx.doi.org/10.1016/j.optom.2013.12.001] [PMID: 25000866] 
[10] Arora R, Gupta D, Goyal JL, Jain P. Results of corneal collagen cross-linking in pediatric patients. J Refract Surg 2012; $28(11)$ : 759-62. [http://dx.doi.org/10.3928/1081597X-20121011-02] [PMID: 23347368]

[11] Caporossi A, Mazzotta C, Baiocchi S, Caporossi T, Denaro R, Balestrazzi A. Riboflavin-UVA-induced corneal collagen cross-linking in pediatric patients. Cornea 2012; 31(3): 227-31. [http://dx.doi.org/10.1097/ICO.0b013e31822159f6] [PMID: 22420024]

[12] Vinciguerra P, Albé E, Frueh BE, Trazza S, Epstein D. Two-year corneal cross-linking results in patients younger than 18 years with documented progressive keratoconus. Am J Ophthalmol 2012; 154(3): 520-6. [http://dx.doi.org/10.1016/j.ajo.2012.03.020] [PMID: 22633357]

[13] Chatzis N, Hafezi F. Progression of keratoconus and efficacy of pediatric [corrected] corneal collagen cross-linking in children and adolescents. J Refract Surg 2012; 28(11): 753-8. [http://dx.doi.org/10.3928/1081597X-20121011-01] [PMID: 23347367]

[14] Kankariya VP, Kymionis GD, Diakonis VF, Yoo SH. Management of pediatric keratoconus - evolving role of corneal collagen cross-linking: An update. Indian J Ophthalmol 2013; 61(8): 435-40. [http://dx.doi.org/10.4103/0301-4738.116070] [PMID: 23925333]

[15] Caporossi A, Mazzotta C, Baiocchi S, Caporossi T. Long-term results of riboflavin ultraviolet a corneal collagen cross-linking for keratoconus in Italy: the Siena eye cross study. Am J Ophthalmol 2010; 149(4): 585-93. [http://dx.doi.org/10.1016/j.ajo.2009.10.021] [PMID: 20138607]

[16] Raiskup-Wolf F, Hoyer A, Spoerl E, Pillunat LE. Collagen crosslinking with riboflavin and ultraviolet-A light in keratoconus: Long-term results. J Cataract Refract Surg 2008; 34(5): 796-801. [http://dx.doi.org/10.1016/j.jcrs.2007.12.039] [PMID: 18471635]

[17] Wittig-Silva C, Whiting M, Lamoureux E, Lindsay RG, Sullivan LJ, Snibson GR. A randomized controlled trial of corneal collagen crosslinking in progressive keratoconus: preliminary results. J Refract Surg 2008; 24(7): S720-5. [PMID: 18811118]

[18] Gkika M, Labiris G, Kozobolis V. Corneal collagen cross-linking using riboflavin and ultraviolet-A irradiation: A review of clinical and experimental studies. Int Ophthalmol 2011; 31(4): 309-19. [http://dx.doi.org/10.1007/s10792-011-9460-x] [PMID: 21847678]

[19] Alhayek A, Lu PR. Corneal collagen crosslinking in keratoconus and other eye disease. Int J Ophthalmol 2015; 8(2): 407-18. [PMID: 25938065]

[20] Chan CC, Sharma M, Wachler BS. Effect of inferior-segment Intacs with and without C3-R on keratoconus. J Cataract Refract Surg 2007; 33(1): 75-80. [http://dx.doi.org/10.1016/j.jcrs.2006.09.012] [PMID: 17189797]

[21] Samaras KE, Lake DB. Corneal collagen cross linking (CXL): A review. Int Ophthalmol Clin 2010; 50(3): 89-100. [http://dx.doi.org/10.1097/IIO.0b013e3181e20df6] [PMID: 20611020]

[22] Kanellopoulos AJ. Collagen cross-linking in early keratoconus with riboflavin in a femtosecond laser-created pocket: initial clinical results. J Refract Surg 2009; 25(11): 1034-7. [http://dx.doi.org/10.3928/1081597X-20090901-02] [PMID: 19731884]

[23] Magli A, Forte R, Tortori A, Capasso L, Marsico G, Piozzi E. Epithelium-off corneal collagen cross-linking versus transepithelial crosslinking for pediatric keratoconus. Cornea 2013; 32(5): 597-601. [http://dx.doi.org/10.1097/ICO.0b013e31826cf32d] [PMID: 23132450]

[24] Filippello M, Stagni E, O’Brart D. Transepithelial corneal collagen crosslinking: bilateral study. J Cataract Refract Surg 2012; 38(2): 283-91 [http://dx.doi.org/10.1016/j.jcrs.2011.08.030] [PMID: 22104644]

[25] Koppen C, Wouters K, Mathysen D, Rozema J, Tassignon MJ. Refractive and topographic results of benzalkonium chloride-assisted transepithelial crosslinking. J Cataract Refract Surg 2012; 38(6): 1000-5. [http://dx.doi.org/10.1016/j.jcrs.2012.01.024] [PMID: 22624899]

[26] Leccisotti A, Islam T. Transepithelial corneal collagen cross-linking in keratoconus. J Refract Surg 2010; 26(12): 942-8. [http://dx.doi.org/10.3928/1081597X-20100212-09] [PMID: 20166621]

[27] Buzzonetti L, Petrocelli G. Transepithelial corneal cross-linking in pediatric patients: early results. J Refract Surg 2012; $28(11)$ : 763-7. [http://dx.doi.org/10.3928/1081597X-20121011-03] [PMID: 23347369]

[28] Cinar Y, Cingü AK, Türkcü FM, et al. Comparison of accelerated and conventional corneal collagen cross-linking for progressive keratoconus. Cutan Ocul Toxicol 2014; 33(3): 218-22. [http://dx.doi.org/10.3109/15569527.2013.834497] [PMID: 24147938]

[29] Kanellopoulos AJ. Long term results of a prospective randomized bilateral eye comparison trial of higher fluence, shorter duration ultraviolet A radiation, and riboflavin collagen cross linking for progressive keratoconus. Clin Ophthalmol 2012; 6: 97-101. [http://dx.doi.org/10.2147/OPTH.S27170] [PMID: 22275813]

[30] Leoni-Mesplie S, Mortemousque B, Touboul D, et al. Scalability and severity of keratoconus in children. Am J Ophthalmol 2012; 154: 56-62 [http://dx.doi.org/10.1016/j.ajo.2012.01.025] 
[31] Al Suhaibani AH, Al-Rajhi AA, Al-Motowa S, Wagoner MD. Inverse relationship between age and severity and sequelae of acute corneal hydrops associated with keratoconus. Br J Ophthalmol 2007; 91(7): 984-5. [http://dx.doi.org/10.1136/bjo.2005.085878] [PMID: 17576720]

[32] Li X, Yang H, Rabinowitz YS. Longitudinal study of keratoconus progression. Exp Eye Res 2007; 85(4): $502-7$. [http://dx.doi.org/10.1016/j.exer.2007.06.016] [PMID: 17681291]

[33] Ertan A, Muftuoglu O. Keratoconus clinical findings according to different age and gender groups. Cornea 2008; 27(10): 1109-13. [http://dx.doi.org/10.1097/ICO.0b013e31817f815a] [PMID: 19034122]

[34] Reeves SW, Stinnett S, Adelman RA, Afshari NA. Risk factors for progression to penetrating keratoplasty in patients with keratoconus. Am J Ophthalmol 2005; 140(4): 607-11. [http://dx.doi.org/10.1016/j.ajo.2005.05.029] [PMID: 16226512]

[35] Kamiya K, Shimizu K, Ohmoto F. Effect of aging on corneal biomechanical parameters using the ocular response analyzer. J Refract Surg 2009; 25(10): 888-93. [http://dx.doi.org/10.3928/1081597X-20090917-10] [PMID: 19835329]

[36] Vanathi M, Panda A, Vengayil S, Chaudhuri Z, Dada T. Pediatric keratoplasty. Surv Ophthalmol 2009; 54(2): $245-71$. [http://dx.doi.org/10.1016/j.survophthal.2008.12.011] [PMID: 19298903]

[37] Zotta PG, Moschou KA, Diakonis VF, et al. Corneal collagen cross-linking for progressive keratoconus in pediatric patients: A feasibility study. J Refract Surg 2012; 28(11): 793-9. [http://dx.doi.org/10.3928/1081597X-20121011-08] [PMID: 23347374]

[38] Salman AG. Transepithelial corneal collagen crosslinking for progressive keratoconus in a pediatric age group. J Cataract Refract Surg 2013; 39(8): 1164-70. [http://dx.doi.org/10.1016/j.jcrs.2013.03.017] [PMID: 23790531]

[39] McAnena L, O'Keefe M. Corneal collagen crosslinking in children with keratoconus. J AAPOS 2015; 19(3): $228-32$. [http://dx.doi.org/10.1016/j.jaapos.2015.02.010] [PMID: 26059667]

(C) 2017 Panos et al.

This is an open access article distributed under the terms of the Creative Commons Attribution 4.0 International Public License (CC-BY 4.0), a copy of which is available at: https://creativecommons.org/licenses/by/4.0/legalcode. This license permits unrestricted use, distribution, and reproduction in any medium, provided the original author and source are credited. 